VOL. $18(1978), 161-168$.

\title{
A note on generalised linear complementarity problems
}

\section{J. Parida and B. Sahoo}

Given an $n \times n$ matrix $A$, an n-dimensional vector $q$, and a closed, convex cone $S$ of $k^{n}$, the generalized linear complementarity problem considered here is the following: find a $z \in R^{n}$ such that

$$
\begin{gathered}
A z-q \in S^{*}, z \in S, \\
(A z-q, z)=0,
\end{gathered}
$$

where $S^{*}$ is the polar cone of $S$. The existence of a solution to this problem for arbitrary vector $q$ has been established both analytically and constructively for several classes of matrices $A$. In this note, a new class of matrices, denoted by $J$, is introduced. $A$ is a $J$-matrix if

$$
A z \in S^{*}, z^{T} A z \leq 0, \quad z \in S \text { imply that } z=0 .
$$

The new class can be seen to be broader than previously studied classes. We analytically show that for any $A$ in this class, a solution to the above problem exists for arbitrary vector $q$. This is achieved by using a result on variational inequalities.

\section{Introduction}

The generalized linear complementarity problem is to find a $z \in R^{n}$ satisfying

Received 11 November 1977. 


$$
\begin{gathered}
A z-q \in S^{*}, \quad z \in S, \\
(A z-q, z)=0,
\end{gathered}
$$

where $A$ is a given $n \times n$ matrix, $q$ is a given $n$-dimensional vector, $S$ is a closed, convex cone in $R^{n}$, and $S^{*}$ is the polar cone of $S$.

For $S=R_{+}^{n}$, the complementarity problem (1.1) has been extensively studied in the literature. The existence of a unique solution to this problem has been shown by Dantzig, Cottle [2] for P-matrices, which include all the previously studied matrices for which there is a unique solution. Karamardian [7] has solved this problem for the class of regular matrices, and thus has enlarged the class of matrices each of which guarantees a solution (but not necessarily unique).

Habetler and Price [4] have shown that the problem (1.1) has a solution when $S$ is a pointed, closed, convex cone with nonempty interior, $A$ is a strictly $S$-copositive matrix, and either

(i) $q \in$ int $S^{*}$ ur

(ii) $S \subset S^{*}$.

Karamardian [6] has generalized this result, and shown that strict $S$-copositiveness of $A$ is sufficient to ensure the existence of a solution to (1.1). In [9], the authors have shown that the linear complementarity problem defined over polyhedral cones in a complex $n$-space possesses a solution when $A$ is a strictly $S$-copositive complex matrix.

In this note, we define a new class $J$ of matrices $A$ such that: if $A \in J$, then

$$
A z \in S^{*}, z^{T} A z \leq 0, z \in S \text { imply that } z=0 .
$$

The classes of $P$ - and regular matrices become proper subclasses of this class when $S$ is taken as $R_{+}^{n}$. It is also found that the class $J$ includes the class of strictly $S$-copositive matrices, and thus becomes a broader class than previously studied ones.

We show that if $A \in J$ and there exists a vector $p \in$ int $S^{*}$ such that the system $0 \neq z \in S, A z+p \in S^{*}, z^{T}(A z+p)=0$ is not consistent, then (1.1) possesses a solution for every vector $q \in R^{n}$. 


\section{Notations and definitions}

Throughout this note, $R^{n}$ will denote euclidean $n$-space with the usual inner product $\langle x, y\rangle=y^{T} x$ of $x, y \in R^{n}$ and norm $\|x\|$ of $x \in R^{n} \cdot R_{+}^{n}$ denotes the nonnegative orthant of $R^{n}$. A subset $S$ of $R^{n}$ will be called a closed, convex cone if, and only if,

(i) $S$ is closed, and

(ii) $\alpha x+\beta y \in S$ for $\alpha, \beta \geq 0$ and $x, y \in S$.

The polar of a cone $S$ is the cone $S^{*}$ defined by

$$
S^{*}=\left\{x \in R^{n}:\langle x, y\rangle \geq 0 \text { for all } y \in S\right\} \text {. }
$$

The interior of $S^{*}$ is given by

$$
\text { int } S^{*}=\left\{x \in S^{*}:\langle x, y\rangle>0 \text { for all } 0 \neq y \in S\right\} \text {. }
$$

A cone is said to be pointed if whenever $x \neq 0$ is in the cone, $-x$ is not in the cone. For a closed, convex cone $S$, int $S^{*}$ is nonempty if, and only if, $S$ is pointed.

A square matrix $A$ is a $P$-matrix if all its principal minors are positive.

For every $x \geq 0$, let $I_{+}(x)$ and $I_{0}(x)$ denote the set of indices corresponding to the positive and zero components of $x$; that is, $I_{+}(x)=\left\{i: x_{i}>0\right\}$ and $I_{0}(x)=\left\{i: x_{i}=0\right\}$. A square matrix $A$ is said to be regular if the system

$$
\begin{gathered}
(A x)_{i}+t=0 \text { for } i \in I_{+}(x), \\
(A x)_{i}+t \geq 0 \text { for } i \in I_{0}(x), \\
0 \neq x \geq 0, t \geq 0,
\end{gathered}
$$

is inconsistent. Here $(A x)_{i}$ denotes the $i$ th component of the vector $A x$.

A square matrix $A$ is said to be strictly $S$-copositive if $x^{T} A x>0$ for all $0 \neq x \in S$. 


\section{Preliminary results}

LEMMA 3.1. Let $A$ be an $n \times n$ matrix, and let $S$ be a closed, convex cone in $R^{n}$.

(a) If $A$ is strictly S-copositive, then $A \in J$.

(b) If $S=R_{+}^{n}$, then $A$ is in $J$ whenever $A$ is either

(i) a P-matrix or

(ii) a regular matrix.

Proof. (a) It immediately follows from the definitions of $J$ - and $S$-copositive matrices given above.

(b) Let $A$ be a $P$-matrix. The conclusion (b) for $P$-matrices follows from the following result of Fiedler and Pták [3]: if $A$ is a $P$-matrix, then for each $0 \neq x \in R^{n}$, there is an index $i$ for which $x_{i}(A x)_{i}>0$.

To prove the second part of $(b)$, we observe that when $S=R_{+}^{n}$, the system $x \in S, A x \in S^{*}, x^{T} A x \leq 0$, reduces to $x \geq 0, A x \geq 0$, $x^{T} A x \leq 0$, the consistency of which implies that $x_{i}(A x)_{i}=0$ for $1 \leq i \leq n$. If $x \neq 0$, we will have $(A x)_{i}=0$ for $i \in I_{+}(x)$ and $(A x)_{i} \geq 0$ for $i \in I_{0}(x)$, which is a contradiction to the regularity of $A$.

REMARK 3.2. It is interesting to note that the class of regular matrices is properly included in $J$. For example, the matrix $\left(\begin{array}{cc}-2 & 2 \\ -1 & 2\end{array}\right)$ is a $J$-matrix, but not regular.

LEMMA 3.3. Let $C$ be a closed, convex cone in $R^{n}$ with nonempty interior, $d \in R^{n}$, and let $x \in$ int $C$. Then there is $a \lambda_{0}>0$ such that $\lambda x+d \in C$ for every $\lambda \geq \lambda_{0}$.

Proof. Since $x \in$ int $C$, there is a $\delta>0$ such that $u \in C$ whenever $\|u-x\| \leq \delta$. Consider the vector $w=x+d / \mu$ for some $\mu>0$. Now 
$\|\omega-x\|=\|d\| / \mu \leq \delta$ if $\mu \geq\|d\| / \delta$. Taking $\lambda_{0}=\|d\| / \delta$, we see that $x+d / \lambda \in C$ for every $\lambda \geq \lambda_{0}$. Since $C$ is a cone, $\lambda x+d=\lambda(x+(d / \lambda))$ will be in $C$ for all $\lambda \geq \lambda_{0}$.

LEMMA 3.4. Let $c$ be a closed, convex cone in $R^{n}$ with nonempty interior, $d \in C$, and let $x \in$ int $C$. Then $x+d \in$ int $C$.

proof. If $x, d \in C$, then $x+d \in C$ because $C$ is a convex cone. Further, if $0 \neq y \in C^{*}$, then we have $\left.\langle d, y\rangle \geq 0,\langle x, y\rangle\right\rangle 0$, and hence $\langle x+d, y\rangle>0$, from which it follows that $(x+d)$ is in int $C$.

We shall make use of the following results.

LEMMA 3.5 [4, Lemma 5.1, p. 227]. Let $S$ be a pointed, closed, convex cone in $R^{n}$, and let $p \in$ int $S^{*}$. Then the set

$$
V=\{x: x \in S,\langle p, x\rangle=1\}
$$

is bounded.

THEOREM 3.6. If $F: R^{n} \rightarrow R^{n}$ is a contiruous mapping on the nonempty, compact, convex set $C$ in $R^{n}$, then there is an $x^{0}$ in $C$ such that

$$
\left\langle F\left(x^{0}\right), x-x^{0}\right\rangle \geq 0 \text { for all } x \in C \text {. }
$$

REMARK 3.7. Theorem 3.6 was first stated and proved in [5]. A complex version of this result has been used by the present authors to obtain some existence theorems for nonlinear complementarity problems in complex space [8].

\section{Solvability of the complementarity problem}

THEOREM 4.1. Let $S$ be a pointed, closed, convex cone in $R^{n}$. If $A \in J$ and there exists a vector $p \in$ int $S^{*}$ such that the system $0 \neq z \in S, A z+p \in S^{*}, z^{T}(A z+p)=0$ is not consistent, then for each $q \in R^{n}$ there is a vector $z^{0}$ satisfying $A z-q \in S^{*}, z \in S$.

Proof. Consider the set $V=\{z: z \in S,(p, z)=1\}$. It is clear that $V$ is a closed, convex set, and by Lemma 3.5 , it is also bounded. Thus $V$ is a nonempty, compact, convex set in $R^{n}$. Now applying Theorem 
3.6, we get a point $\bar{z}$ in $V$ such that

$$
\langle\bar{A} \bar{z}, z-\bar{z}\rangle \geq 0 \text { for all } z \in V \text {. }
$$

It follows from (4.1) that

$$
\langle A \bar{z}, \bar{z}\rangle=\min _{z \in V}\langle A \bar{z}, z\rangle,
$$

and therefore, the following set of necessary conditions [1] is satisfied:

$$
\begin{aligned}
& A \bar{z}+n p \in S^{*},(A \bar{z}+n p, \bar{z}\rangle=0, \\
& \bar{z} \in S,\langle p, \bar{z}\rangle=1, \eta \in R .
\end{aligned}
$$

Obviously $\bar{z} \neq 0$. Now the consistency of (4.2) for a vector $0 \neq \bar{z} \in S$ and $n \geq 0$ will contradict the assumptions made in the statement of the theorem. So $n<0$, and thus we have a $0 \neq y=\bar{z} / n \in S$ satisfying Ay $-p \in S^{*}$. Since $p \in$ int $S^{*}$, it follows from Lemma 3.4 that

$A y \in$ int $S^{*}$. Now, for any given vector $q \in R^{n}$, Lemma 3.3 will determine a $\lambda>0$ such that $\lambda(A y)-q \in S^{*}$. Since $S$ is a cone, $\lambda y \in S$. The proof of the theorem is then completed by writing $z^{0}=\lambda y$.

Now we give the following existence theorem.

THEOREM 4.2. Let $S$ be a pointed, closed, convex cone in $R^{n}$, and let $p$ be a vector in int $S^{*}$ such that the system $0 \neq z \in S$, $A z+p \in S^{*}, z^{T}(A z+p)=0$ is inconsistent. Then there is a solution to (1.1) for each $q \in R^{n}$ if $A$ is a J-matrix.

Proof. Consider the function

$$
F(z, t)=\left[\begin{array}{c}
A z+t(p-q) \\
t
\end{array}\right]
$$

defined over the set

$$
C=\{(z, t): z \in S, t \geq 0,\langle p, z\rangle+t=1\} .
$$

From Lemma 3.5 , it is clear that $C$ is a nonempty, compact, convex set in $R^{n+1}$. It is also evident that $F(z, t)$ and $C$ satisfy the conditions of Theorem 3.6, and hence, there exists a point $(\bar{z}, \bar{t})$ in $C$ such that

$$
(A \bar{z}+\bar{t}(p-q), z-\bar{z})+\bar{t}(t-\bar{t}) \geq 0 \text { for all }(z, t) \in C \text {. }
$$

But this means that 


$$
\langle A \bar{z}+\bar{t}(p-q), \bar{z}\rangle+\bar{t} \cdot \bar{t}=\min _{(z, t) \in C}(\langle A \bar{z}+\bar{t}(p-q), z\rangle+\bar{t} \cdot t) .
$$

Now using the Kuhn-Tucker necessary conditions of optimality [1] for cone domains, we have a $\zeta_{0}$ in $R$ such that

$$
\begin{gathered}
A \bar{z}+\bar{t}(p-q)+\zeta_{0} p \in S^{*}, \bar{t}+\zeta_{0} \geq 0, \\
\left\langle A \bar{z}+\bar{t}(p-q)+\zeta_{0} p, \bar{z}\right\rangle=0, \bar{t}\left(\bar{t}+\zeta_{0}\right)=0 \\
\bar{z} \in S, \bar{t} \geq 0,\langle p, \bar{z}\rangle+\bar{t}=1 .
\end{gathered}
$$

Suppose that $\bar{t}=0$. Since $\langle p, \bar{z}\rangle+\bar{t}=1$ and $p \in$ int $S^{*}, \bar{z} \neq 0$. If this is the case, then (4.3) will imply that the system

$$
0 \neq \bar{z} \in S, A \bar{z}+\zeta_{0} p \in S^{*} \text {, }
$$

$$
\left\langle A \bar{z}+\zeta_{0} p, \bar{z}\right\rangle=0, \zeta_{0} \geq 0 \text {, }
$$

is consistent. When $\zeta_{0}=0$, the consistency of (4.4) will contradict the assumption that $A$ is a $J$-matrix. Further, when $\zeta_{0}>0,(4.4)$ will yield a nonzero vector $\bar{y}=\bar{z} / \zeta_{0} \in S$ satisfying $A \bar{y}+p \in S^{*}$, $\bar{y}^{T}(A \bar{y}+p)=0$, again a contradiction. Hence, $\bar{t}>0$, and since $\bar{t}\left(\bar{t}+\zeta_{0}\right)=0$, therefore, we have $\bar{t}+\zeta_{0}=0$. Now substituting $\zeta_{0}=-\bar{t}$ in (4.3), and then dividing throughout the resulting relations by $\bar{t}$, we get the desired solution.

REMARK 4.3. We do not require any other assumption for the cone $S$, except that it is to be pointed in the statement of Theorem 4.2. But for $q$, we impose the restriction that when $-q=p \in$ int $S^{*}$, there is no nonzero solution to (1.1). IFor this value of $q$, zero is obviously a solution. This restriction is automatically satisfied when $A$ is strictly $S$-copositive, whereas if $A$ is a regular matrix and $S=R_{+}^{n}$, (1.1) has no nonzero solution for the vector $-q=e t, t \geq 0$, with $e^{T}=(1,1, \ldots, 1)$. 


\section{References}

[1] M.S. Bazaraa and J.J. Goode, "Necessary optimality criteria in mathematical programming in the presence of differentiability", J. Math. Anal. Appl. 40 (1972), 609-621.

[2] G.B. Dantzig, R.W. Cottle, "Positive (semi-) definite programming", Nonlinear programing, 55-73 (North-Holland, Amsterdam; Interscience [John Wiley \& Sons], New York; 1967).

[3] Miroslav Fiedler and Vlastimi I Pták, "On matrices with non-positive off-diagonal elements and positive principal minors", Czechoslavak Math. J. 12 (1962), 382-400.

[4] G.J. Habetler and A.L. Price, "Existence theory for generalized nonlinear complementarity problems", J. Optimization Theory Appl. 7 (1971), 223-239.

[5] Philip Hartman and Guido Stampacchia, "On some non-linear elliptic differential-functional equations", Acta Math. 115 (1966), $271-310$.

[6] S. Karamardian, "Generalized complementarity problem", J. Optimization Theory App 2. 8 (1971), 161-168.

[7] S. Karamardian, "The complementarity problem", Math. Programming 2 (1972), 107-129.

[8] J. Parida and B. Sahoo, "On the complex nonlinear complementarity problem", Bulz. Austral. Math. Soc. 14 (1976), 129-136.

[9] J. Parida and B. Sahoo, "Existence theory for the complex nonlinear complementarity problem", Bulz. Austral. Math. Soc. 14 (1976), 417-423.

Department of Mathematics,

Regional Engineering College,

Rourkela, Orissa,

India. 